\title{
An Integral Equation Method with High-Order Collocation Implementations for Pricing American Put Options
}

\author{
Jingtang Ma \\ School of Economic Mathematics, Southwestern University of Finance and Economics, Chengdu, 611130, China \\ E-mail: mjt@swufe.edu.cn
}

Kaili Xiang

School of Economic Mathematics, Southwestern University of Finance and Economics, Chengdu, 611130, China

E-mail: xiangkl@swufe.edu.cn

Yingjun Jiang

Department of Mathematics and Scientific Computing, Changsha University of Science and Technology

Changsha, 410076, China

E-mail:jiangyingjun@csust.edu.cn

This research was supported by a grant from the "project 211 (phase III)" of Southwestern University of Finance and Economics.

\begin{abstract}
The aim of this paper is to solve a free boundary problem arising in pricing American put options. It is known that the free boundary (optimal exercise boundary) satisfies a "nonstandard" Volterra integral equation. This Volterra integral equation is resolved by a high-order collocation method based on graded meshes. With the computed free boundary, a Black-Scholes equation for pricing the American put options is solved by a moving mesh method. Numerical examples are provided to confirm the efficiency of the approach.
\end{abstract}

Keywords: American put options, Optimal exercise boundary, Collocation methods, Integral equations

\section{Introduction}

Assume that $S$ is the underlying asset price, $t$ is the time, and $V=V(S, t)$ is the put option value. Let $r$ denote the risk-free interest rate, $\sigma$ the volatility of the underlying asset price, and $q$ the continuous dividend yield. Then $V$ satisfies the following free boundary problem (see e.g., in Hull (2007), Jiang (2005) for the derivation)

$$
\begin{aligned}
& \frac{\partial V}{\partial t}+\frac{\sigma^{2}}{2} S^{2} \frac{\partial^{2} V}{\partial S^{2}}+(r-q) S \frac{\partial V}{\partial S}-r V=0, S_{f}(t) \leq S<+\infty, 0 \leq t \leq T, \\
& V\left(S_{f}(t), t\right)=K-S_{f}(t), \\
& \frac{\partial V\left(S_{f}(t), t\right)}{\partial S}=-1, \\
& V \rightarrow 0, \text { as } S \rightarrow+\infty, \\
& V(S, T)=\max (K-S, 0), \\
& S_{f}(T)=\min \left(\frac{r K}{q}, K\right),
\end{aligned}
$$

where $S_{f}(t)$ is the optimal exercise boundary (free boundary), $T$ is the expiry time $K$ is the strike price, and $V(S, T)$ is called payoff function. It has several difficulties to solve the above free boundary value problem. Being coupled with the unknown solution $V$, the free boundary satisfies condition (3) which is a highly nonlinear equation. So it is indeed not an easy task to get a highly accurate resolution to the free boundary. Moreover since numerical methods cannot be applied directly to the unbounded domain, a feasible artificial boundary(or artificial boundary condition) needs to be set up. In addition the non-smoothness of $V$, which belongs to $H^{1}$, affects the accuracy of a numerical method. Therefore a kind of selfadaptive methods is required to improve the accuracy of solving theBlack-Scholes equation. Among the vast of literatures on the numerical solution of the above free boundary value 
problems, Han and $\mathrm{Wu}$ (2003) derive an artificial boundary condition for the heat equation arising from the transformation of the Black-Scholes equation in an American call option. Wong and Zhao (2008) derive artificial boundary conditions for a CEV model with Black-Scholes model as a special case. Tangman et al. (2008) provide a survey to the numerical methods for pricing American options and develop a new finite difference method to deal with the singularity existing at the strike price in the payoff function which deceases the accuracy of the solution. Fusai et al. (2007) investigate quadrature method for solving the free boundary problem.

Cox (1979) (compare with Jiang (2005) and Robinstein (1991)) proves that the free boundary satisfies a "nonstandard" Volterra integral equation which is very hard to solve (see in Brunner (2004)). The "nonstandard" Volterra integral equation is given by

$$
\begin{aligned}
S_{f}(t) & =K+S_{f}(t) e^{-q(T-t)} N\left(-\frac{\ln \frac{S_{f}(t)}{K}+\beta_{2}(T-t)}{\sigma \sqrt{T-t}}\right) \\
& -K e^{-r(T-t)} N\left(-\frac{\ln \frac{S_{f}(t)}{K}+\beta_{1}(T-t)}{\sigma \sqrt{T-t}}\right) \\
& +q S_{f}(t) \int_{t}^{T} e^{-q(\eta-t)}\left[1-N\left(-\frac{\ln \frac{S_{f}(t)}{S_{f}(\eta)}+\beta_{2}(\eta-t)}{\sigma \sqrt{\eta-t}}\right)\right] d \eta \\
& -r K \int_{t}^{T} e^{-r(\eta-t)}\left[1-N\left(-\frac{\ln \frac{S_{f}(t)}{S_{f}(\eta)}+\beta_{1}(\eta-t)}{\sigma \sqrt{\eta-t}}\right)\right] d \eta
\end{aligned}
$$

with $S_{f}(T)=\min \left(\frac{r K}{q}, K\right)$, where $N(x)=\frac{1}{\sqrt{2 \pi}} \int_{-\infty}^{x} e^{-\frac{s^{2}}{2}} d s$, and $\beta_{1}=r-q-\frac{\sigma^{2}}{2} ; \beta_{2}=r-q+\frac{\sigma^{2}}{2}$.

In this paper, we resolve the free boundary by solving the "nonstandard" Volterra integral equation (7). A high-order collocation method is developed to solve the "nonstandard" Volterra integral equation. Moreover the solution of the "nonstandard" Volterra integral equation is singular. Therefore the graded meshes, which is introduced by Brunner (1985) (see also in Brunner (2004)), are used in the collocation methods. Since the solution $V$ is not globally smooth, a moving mesh method is the best choice to solve the Black-Scholes equation.

In the history, a few of works, cf. Huang et al. (1996), Ju (1998), Detemple and Tian (2002) have studied the implementations of the integral equations methods for pricing the American put options. However their approaches for solving the integral equations are based on low-order approximations and the numerical quadratures are used to evaluate the EEP (Early Exercise Premium) representation of the option price (see e.g., in Detemple (2002)). While to our knowledge, in order to get highly accurate numerical solutions of the underlying PDEs, it is rather necessary to resolve the free boundary with high accuracy.

In the next section the high-order collocation methods are described to solve the "nonstandard" Volterra integral equations that the free boundary function satisfies; In Section 3, the moving mesh method is introduced to solve the Black-Scholes equations; In Section 4 a variety of numerical examples are carried out to confirm the efficiency of the method; In the final section conclusions are given.

\section{Collocation methods for "nonstandard" Volterra integral equations}

Now we solve the "nonstandard" Volterra integral equation (7). To do that we transform the equation into the following form by replacing $t$ with $T-t$, and then using $\tilde{S}_{f}(t)=S_{f}(T-t)$ and the fact that $1-N(x)=N(-x)$

$$
\begin{aligned}
\tilde{S}_{f}(t) & =K+\tilde{S}_{f}(t) e^{-q t} N\left(-\frac{\ln \frac{\tilde{S}_{f}(t)}{K}+\beta_{2} t}{\sigma \sqrt{t}}\right) \\
& -K e^{-r t} N\left(-\frac{\ln \frac{\tilde{S}_{f}(t)}{K}+\beta_{1} t}{\sigma \sqrt{t}}\right)
\end{aligned}
$$




$$
\begin{aligned}
+q \tilde{S}_{f}(t) \int_{0}^{t} e^{-q(t-\tau)}\left[1-N\left(-\frac{\ln \frac{\tilde{s}_{f(t)}}{\tilde{S}_{f(\tau)}}+\beta_{2}(t-\tau)}{\sigma \sqrt{t-\tau}}\right)\right] d \tau \\
\quad-r K \int_{0}^{t} e^{-r(t-\tau)}\left[1-N\left(-\frac{\ln \frac{\tilde{S}_{f}(t)}{\tilde{S}_{f}(\tau)}+\beta_{1}(t-\tau)}{\sigma \sqrt{t-\tau}}\right)\right] d \tau
\end{aligned}
$$

with $\tilde{S}_{f}(0)=\min \left(\frac{r K}{q}, K\right)$.

The asymptotic properties of the solutions to the above "nonstandard" Volterra integral equation (7) (or the equivalent form (8) are studied e.g., in Cox et al. (1979), Robinstein (1991), Jiang (2005)). We summarize the results into the following theorem.

Theorem 2.1 If $0<t<<1$, then the solution to (8) has the following asymptotic results.

(i) For $q<r$, it holds that

$$
\frac{K-\tilde{S_{f}}(t)}{K} \approx \sigma \sqrt{t|\ln t|}
$$

(ii) For $q=r$, it holds that

$$
\frac{K-\tilde{S_{f}}(t)}{K} \approx \sqrt{2} \sigma \sqrt{t|\ln t|}
$$

(iii) For $q>r$, it holds that

$$
\frac{\frac{r K}{q}-S_{f}(t)}{\frac{r K}{q}} \approx \frac{\sigma}{\sqrt{2}} \alpha \sqrt{t} ;
$$

where $\alpha$ is the solution of

$$
\alpha^{3} e^{\frac{\alpha^{2}}{4} \int_{\alpha}^{\infty} e^{-\frac{s^{2}}{4}}} d s=2\left(2-\alpha^{2}\right) .
$$

It is seen from the theorem that the derivatives of $\tilde{S_{f}}$ are singular at the origin. Thus the use of uniform meshes cannot give an optimal accuracy in the numerical solution of equation (8). Therefore the graded meshes, which is developed in Brunner (1985) (cf. Brunner (2004)), with mesh points satisfying $t_{i}=T\left(\frac{i}{L}\right)^{2}, \quad i=0,1, \ldots, L$ are used in finding the numerical solution of equation (8) (or (7)). Now we describe the high-order collocation methods for equation (8) (or (7)). To do that we rewrite equation (8) into a more feasible form using the transformation $y(t)=\ln \tilde{S_{f}}(t)$,

$$
\begin{gathered}
e^{y(t)}=K+e^{y(t)-q t} N\left(-\frac{y(t)-\ln K+\beta_{2} t}{\sigma \sqrt{t}}\right)-K e^{-r t} N\left(-\frac{y(t)-\ln K+\beta_{1} t}{\sigma \sqrt{t}}\right) \\
+q e^{y(t)} \int_{0}^{t} e^{-q(t-\tau)} N\left(-\frac{y(t)-y(\tau)+\beta_{2}(t-\tau)}{\sigma \sqrt{t-\tau}}\right) d \tau \\
-r K \int_{0}^{t} e^{-r(t-\tau)} N\left(-\frac{y(t)-y(\tau)+\beta_{1}(t-\tau)}{\sigma \sqrt{t-\tau}}\right) d \tau .
\end{gathered}
$$

Define a piecewise polynomial space $\Pi_{3}^{-1}(0, T]=\left\{p: p(t) \in P_{3}, t \in\left(t_{i}, t_{i+1}\right], i=0,1, \ldots, L-1\right\}$,

where $P_{3}$ is the set of polynomials of degree 3. The high-order collocation method is defined by: Find $Y(t) \in \Pi_{3}^{-1}$ 
such that

$$
\begin{gathered}
e^{Y(t)}=K+e^{Y(t)-q t} N\left(-\frac{Y(t)-\ln K+\beta_{2} t}{\sigma \sqrt{t}}\right)-K e^{-r t} N\left(-\frac{Y(t)-\ln K+\beta_{1} t}{\sigma \sqrt{t}}\right) \\
+q e^{Y(t)} \int_{0}^{t} e^{-q(t-\tau)} N\left(-\frac{Y(t)-Y(\tau)+\beta_{2}(t-\tau)}{\sigma \sqrt{t-\tau}}\right) d \tau \\
-r K \int_{0}^{t} e^{-r(t-\tau)} N\left(-\frac{Y(t)-Y(\tau)+\beta_{1}(t-\tau)}{\sigma \sqrt{t-\tau}}\right) d \tau
\end{gathered}
$$

holds exactly at the collocating points

$$
t_{i, j}=t_{i}+\frac{j}{4}\left(t_{i+1}-t_{i}\right), \quad j=1,2,3,4 ; \quad i=0,1, \ldots, L-1
$$

At time interval $\left(t_{i}, t_{i+1}\right]$, polynomial $\mathrm{Y}(\mathrm{t})$ can be written by

$$
Y(t)=\sum_{j=1}^{4} Y_{j}^{i} l_{j}^{i}(t)
$$

where $l_{j}^{i}(t)$ is the Lagrange basis at points $t_{i, j}, j=1,2,3,4$; i.e.,

$$
l_{j}^{i}(t)=\prod_{k \neq j} \frac{t-t_{k}^{i}}{t_{j}^{i}-t_{k}^{i}} .
$$

Inserting (14) into (13) gives the computational form

$$
F_{j}^{i}\left(Y_{1}^{i}, Y_{2}^{i}, Y_{3}^{i}, Y_{4}^{i}\right)=0, \quad j=1,2,3,4 ; \quad i=0,1, \ldots, L-1
$$

for the unknowns $Y_{1}^{i}, Y_{2}^{i}, Y_{3}^{i}, Y_{4}^{i}, \quad i=0,1, \ldots, L-1$

where the function $F_{j}^{i}$ is given by

$$
\mathrm{F}_{\mathrm{j}}^{\mathrm{i}}=e^{Y_{j}^{i}}-K-e^{Y_{j}^{i}-q t_{i, j}} N\left(\varepsilon_{2}\right)+K e^{-r t_{i, j}} N\left(\varepsilon_{1}\right)=q e^{Y_{j}^{i}} \int_{0}^{t_{i, j}} e^{-q\left(t_{i, j}-\tau\right)} N\left(\eta_{2}\right) d \tau+r K \int_{0}^{t_{i, j}} e^{-r\left(t_{i, j}-\tau\right)} N\left(\eta_{1}\right) d \tau,
$$

and

$$
\varepsilon_{2}=-\frac{\mathrm{Y}_{j}^{i}-\ln K+\beta_{2} t_{i, j}}{\sigma \sqrt{t_{i, j}}}, \quad \varepsilon_{1}=-\frac{\mathrm{Y}_{j}^{i}-\ln K+\beta_{1} t_{i, j}}{\sigma \sqrt{t_{i, j}}},
$$

and

$$
\eta_{2}=-\frac{\mathrm{Y}_{j}^{i}-Y(\tau)+\beta_{2}\left(t_{i, j}-\tau\right)}{\sigma \sqrt{t_{i, j}-\tau}}, \quad \eta_{1}=-\frac{\mathrm{Y}_{j}^{i}-Y(\tau)+\beta_{1}\left(t_{i, j}-\tau\right)}{\sigma \sqrt{t_{i, j}-\tau}} .
$$

To solve the nonlinear algebraic equations (15), the Newton's method is used. The Jacobian is calculated as follows:

$$
\begin{aligned}
& \frac{\partial \mathrm{F}_{j}^{i}}{\partial Y_{j}^{i}} \approx e^{Y_{j}^{i}}-e^{Y_{j}^{i}-q t_{i, j}} N\left(\varepsilon_{2}\right) \\
& +\frac{e^{Y_{j}^{i}-q t_{i, j}-\frac{\left(\varepsilon_{2}\right)^{2}}{2}}-K e^{-r t_{i, j}-\frac{\left(\varepsilon_{1}\right)^{2}}{2}}}{\sigma \sqrt{2 \pi t_{i, j}}}-q e^{Y_{j}^{i}} \int_{0}^{t_{i, j}} e^{-q\left(t_{i, j}-\tau\right)} N\left(\eta_{2}\right) d \tau
\end{aligned}
$$

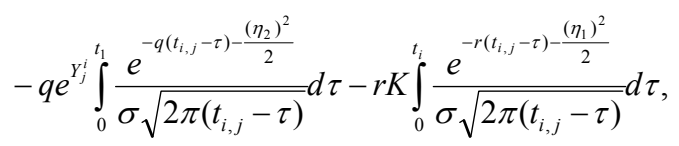

and 


$$
\frac{\partial \mathrm{F}_{j}^{i}}{\partial Y_{k}^{i}} \approx 0, \text { for } k \neq j .
$$

Integrals in (17) are calculated by

$\int_{0}^{t_{i}} \frac{f(\tau)}{\sqrt{t_{i, j}-\tau}}=\int_{j}^{t_{i, j}} \frac{f\left(t_{i, j}-\tau\right)}{\sqrt{t}} d \tau=2 \int_{\sqrt{j} \frac{\Delta_{i+1}}{4}}^{\sqrt{t_{i, j}}} f\left(t_{i, j}-\tau^{2}\right) d \tau$,

where $\Delta t_{i+1}=t_{i+1}-t_{i}$.

Denote $Y^{i}=\left(Y_{1}^{i}, Y_{2}^{i}, Y_{3}^{i}, Y_{4}^{i}\right)^{T}$ and $Z^{(k)}=\left(Z_{1}^{(k)}, Z_{2}^{(k)}, Z_{3}^{(k)}, Z_{4}^{(k)}\right)^{T}$.

Then the Newton's method is given by

$$
Z_{j}^{(k+1)}=Z_{j}^{(k)}-\frac{F_{j}^{i}\left(Z^{(k)}\right)}{\lambda^{(k)}}, \quad j=1,2,3,4 ; \quad k=0,1, \ldots, M,
$$

where

$$
\lambda^{(k)}=\max _{1 \leq j \leq 4} \frac{\partial F_{j}^{i}\left(Z^{(k)}\right)}{\partial Z_{j}^{(k)}} .
$$

The initial value $Z^{(0)}$ is taken as the extrapolation of $Y^{i-1}$ at the interval $\left(t_{i}, t_{i+1}\right]$. The stopping value of Newton's method gives the solution at interval $\left(t_{i}, t_{i+1}\right]$, i.e., $Y^{i}=Z^{(M)}$. All the involved integrals are approximated by four-points Gauss-Legendre quadrature. The following polynomial approximation of the standard normal distribution function with six digital accuracy is given by (see e.g., in Hull (2007))

$$
N(x)=\begin{array}{cc}
1-N^{\prime}(x)\left(a_{1} k+a_{2} k^{2}+a_{3} k^{3}+a_{4} k^{4}+a_{5} k^{5}\right), & \begin{array}{c}
x \geq 0 \\
1-N(-x),
\end{array} \\
x<0,
\end{array}
$$

where

$k=\frac{1}{1+\gamma x}, \quad \gamma=0.2316419$,

$a_{1}=0.319381530, a_{2}=-0.356563782, a_{3}=1.781477937$,

$a_{4}=-1.821255978, a_{5}=1.330274429$,

and

$N^{\prime}(x)=\frac{1}{\sqrt{2 \pi}} e^{-x^{2} / 2}$.

The above computational approach gives that $Y(t)=y(t)=\ln \tilde{S}_{f}(t)$.

Thus

$$
\tilde{S}_{f}(t) \approx e^{Y(t)}
$$

So the optimal exercise boundary is given by $S_{f}(t)=\tilde{S}_{f}(T-t) \approx e^{Y(T-t)}$.

\section{Moving mesh method for Black-Scholes equations}

In this section we solve the Black-Scholes equation (1) with conditions (2),(4),(5). From the derivation of the "nonstandard" Volterra integral equation that the free boundary (optimal exercise boundary) satisfies (cf. Jiang (2005)), we can easily verify that the solution of (7) satisfies condition (3) and (6). This fact will also be confirmed by the numerical tests in the next section.

For the ease of computation, the backward problem - (1), (2), (4), (5) is transformed into a forward problem of the following form by $\tilde{\mathrm{V}}(S, t)=V(S, T-t)$

$$
\frac{\partial \tilde{V}}{\partial t}-\frac{\sigma^{2}}{2} S^{2} \frac{\partial^{2} \tilde{V}}{\partial S^{2}}-(r-q) S \frac{\partial \tilde{V}}{\partial S}+r \tilde{V}=0, \quad \tilde{S}_{f}(t) \leq S \leq+\infty, \quad 0 \leq t \leq T,
$$


with the following conditions

$\tilde{V}\left(\tilde{S}_{f}(t), t\right)=K-\tilde{S}_{f}(t), \quad \tilde{V}(+\infty, t)=0, \tilde{V}(S, t)=\max (K-S, 0)$.

To proceed the computation, we need to truncate the unbounded domain into a bounded domain. According to the asymptotic properties of the analytical solution, we set up a time-dependent artificial boundary to ensure the condition $\tilde{V}(R(t), t) \approx 0$. We take the time-dependent artificial boundary as the form

$$
R(t)=a_{0} l_{0}(t)+a_{1} l_{1}(t)+\gamma\left(K-\tilde{S}_{f}(t)\right),
$$

where $l_{1}, l_{2}$ are Lagrange bases associated with two points $t=0, t=T$ and the nonnegative constants $a_{0}, a_{1}, \gamma$ are the user-defined parameters. Therefore we solve the Black-Scholes equation (21) on the bounded domain $\left[S_{f}(t), R(t)\right]$ with conditions

$$
\tilde{V}\left(\tilde{S}_{f}(t), t\right)=K-\tilde{S}_{f}(t), \tilde{V}(R(t), t)=0, \quad \tilde{V}(S, 0)=\max (K-S, 0) .
$$

We use Landau's transformation (see e.g., in Landau (1950))

$$
S(\cdot, t):[0,1] \rightarrow\left[\tilde{S}_{f}(t), R(t)\right],
$$

satisfying

$$
S(\varepsilon, t)=\tilde{S}_{f}(t)+\varepsilon\left(R(t)-\tilde{S}_{f}(t)\right),
$$

to transform equation (21) into the form

$$
\dot{\tilde{V}}-\dot{S} \frac{\partial \tilde{V}}{\partial S}-\frac{\sigma^{2}}{2} S^{2} \frac{\partial^{2} \tilde{V}}{\partial S^{2}}-(r-q) S \frac{\partial \tilde{V}}{\partial S}+r \tilde{V}=0,
$$

where

$$
\dot{\tilde{V}}=\dot{\tilde{V}}(S(\varepsilon, t), t)=\frac{\partial \tilde{V}}{\partial t}+\dot{S} \frac{\partial \tilde{V}}{\partial S},
$$

and

$\dot{\mathrm{S}}=\frac{\partial \mathrm{S}(\varepsilon, t)}{\partial t}$.

Note that the use of fixed mesh for $\varepsilon: 0 \equiv \varepsilon_{0}<\varepsilon_{1}<\cdots<\varepsilon_{N} \equiv 1$

leads to moving meshes (time-dependent meshes) for $\mathrm{S}$

$$
\tilde{S}_{f}(t) \equiv S\left(\varepsilon_{0}, t\right)<S\left(\varepsilon_{1}, t\right)<\cdots<S\left(\varepsilon_{N}, t\right) \equiv R(t) .
$$

Then for a given time mesh $0 \equiv t_{0}<t_{1}<\cdots<t_{n}<t_{n+1}<\cdots$,

the spatial mesh at $t_{n}$ level is $S\left(\varepsilon_{j}, t_{n}\right)$ for $j=0,1, \ldots, N$, which is denoted by $S\left(\varepsilon_{j}, t_{n}\right)=S_{j}^{n}$.

Now we are in a position to describe the computational scheme for the transformed equation (25) with the conditions (23). Let $\tilde{\mathrm{V}}_{j}^{n}$ be the approximation of $\tilde{V}\left(S_{j}^{n}, t_{n}\right)$. Then the computational scheme is

$$
\begin{gathered}
0=\frac{\tilde{\mathrm{V}}_{j}^{n+1}-\tilde{V}_{j}^{n}}{t_{n+1}-t_{n}}-\frac{S_{j}^{n+1}-S_{j}^{n}}{t_{n+1}-t_{n}} \frac{\tilde{V}_{j+1}^{n+1}-\tilde{V}_{j-1}^{n+1}}{S_{j+1}^{n+1}-S_{j-1}^{n+1}}-\frac{\sigma^{2}}{2}\left(S_{j}^{n+1}\right) \frac{\frac{\tilde{V}_{j+1}^{n+1}-\tilde{V}_{j}^{n+1}}{S_{j+1}^{n+1}-S_{j}^{n+1}}-\frac{\tilde{V}_{j}^{n+1}-\tilde{V}_{j-1}^{n+1}}{S_{j}^{n+1}-S_{j-1}^{n+1}}}{\frac{S_{j+1}^{n+1}-S_{j-1}^{n+1}}{2}} \\
-(r-q) S_{j}^{n+1} \frac{\tilde{V}_{j+1}^{n+1}-\tilde{V}_{j-1}^{n+1}}{S_{j+1}^{n+1}-S_{j-1}^{n+1}}+r \tilde{V}_{j}^{n+1}
\end{gathered}
$$

with 


$$
\tilde{V}_{j}^{0}=\max \left(K-S_{j}^{0}, 0\right), \quad \tilde{V}_{0}^{n}=K-S_{0}^{n}, \quad \tilde{V}_{N}^{n}=0
$$

for $j=1, \ldots, N-1, \quad n=0,1, \ldots$.

\section{Numerical examples}

In the tests, we take the artificial boundary as the form (22), i.e.,

$$
R(t)=a_{0} l_{0}(t)+a_{1} l_{1}(t)+\gamma\left(K-\tilde{S}_{f}(t)\right) .
$$

Let $N$ and $L$ be the number of spatial and time mesh elements, respectively. The following graded mesh for $\varepsilon$ is used, $\varepsilon_{j}=\left(\frac{j}{N}\right)^{2}$.

Using (24) the corresponding spatial mesh at $t_{n}$ level is

$$
\mathrm{S}_{j}^{n}=\tilde{S}_{f}\left(t_{n}\right)+\left(\frac{j}{N}\right)^{2}\left(R\left(t_{n}\right)-\tilde{S}_{f}\left(t_{n}\right)\right) .
$$

The free boundary $\tilde{S}_{f}\left(t_{n}\right)$ is computed from Section $2-(20)$. Thus our algorithm contains two parts - (15) and

(20) for computing $\tilde{S}_{f}(t) ;(26)$ and (27) for computing $\tilde{V}(S, t)$. The solutions to the original problems (1)-(6) are given by

$S_{f}(t)=\tilde{S}_{f}(T-t), \quad V(S, t)=\tilde{V}(S, T-t)$.

Example 4.1 Consider the free boundary value problem for pricing American put options (1)-(6) with $r=0.001, q=0.01, \sigma=0.2, K=100, T=10$,

(the case $r<q$ ).

It is shown by Kim (1990) that

$$
\tilde{\mathrm{S}}_{f}(+\infty)=\frac{\rho K}{\rho-1} \text {, where } \rho=\frac{-\left(r-q-0.5 \sigma^{2}\right)-\sqrt{\left(r-q-0.5 \sigma^{2}\right)^{2}+2 \sigma^{2}}}{\sigma^{2}} \text {. }
$$

Therefore in this example, $\tilde{\mathrm{S}}_{f}(+\infty)=3.260090$. The parameters in $(22)$ are taken as $a_{0}=150, \quad a_{1}=900, \quad r=0$.

The results of the tests are obtained with $L=200$ and $N=400$.

Table 1 shows the tendency to the limit value $\tilde{\mathrm{S}}_{f}(+\infty)=3.260090$. The solution of this example is drawn in Fig. 1 . In Fig. 2, the free boundary (optimal exercise boundary) is demonstrated. In Fig. 3, the asymptotic structure of $\tilde{\mathrm{S}}_{f}(t)$ as $t \rightarrow 0$ is tested: It shows that the numerical $\tilde{\mathrm{S}}_{f}(t)$ satisfies the asymptotic result (11). The test is well consistent with the existing analytical results.

In the subsequence, we do the tests for cases $r=q$ and $r>q$ in a similar manner.

Example 4.2 Consider the free boundary value problem for pricing American put options (1)-(6) with $r=0.01, q=0.01, \sigma=0.2, K=100, T=10$, (the case $r=q$ ).

Using formula (28), we calculate $\tilde{\mathrm{S}}_{f}(+\infty)=26.794919$. The parameters in (22) are taken as $a_{0}=101, a_{1}=500, \quad r=5$.

The results of the tests are obtained with $L=200$ and $N=400$.

Table 2 shows the tendency to the limit value $\tilde{\mathrm{S}}_{f}(+\infty)=26.794919$. The solution of this example is drawn in Fig. 4 . In Fig. 5, the free boundary (optimal exercise boundary) is demonstrated. In Fig. 6, the asymptotic structure of $\tilde{\mathrm{S}}_{f}(t)$ 
as $t \rightarrow 0$ is tested: It confirms that the numerical $\tilde{\mathbf{S}}_{f}(t)$ satisfies the asymptotic result (10).

Example 4.3 Consider the free boundary value problem for pricing American put options (1)-(6) with

$r=0.1, q=0.01, \sigma=0.2, K=100, T=10$,

(the case $r>q$ ).

Using formula (28), we calculate $\tilde{\mathrm{S}}_{f}(+\infty)=82.109165$. The parameters in (22) are taken as

$a_{0}=101, a_{1}=300, r=5$.

The results of the tests are obtained with $L=200$ and $N=400$.

Table 3 shows the tendency to the limit value $\tilde{\mathrm{S}}_{f}(+\infty)=82.109165$. The solution of this example is drawn in Fig. 7. In Fig. 8, the free boundary (optimal exercise boundary) is demonstrated. In Fig. 9, the asymptotic structure of $\tilde{\mathrm{S}}_{f}(t)$ as $t \rightarrow 0$ is tested: It verifies that the numerical $\tilde{\mathrm{S}}_{f}(t)$ satisfies the asymptotic result (9).

\section{Conclusions}

In this paper we have developed a high-order collocation method based upon the graded meshes for solving a "nonstandard" Volterra integral equation for the free boundary (optimal exercise boundary) arising in pricing American put options. With the computed free boundary, we also set up a time-dependent artificial boundary so that the Black-Scholes equation for pricing the American put options can be computed on the bounded domain. The moving mesh methods, with the moving meshes generated by the graded meshes on the computational space, are used to solve the Black-Scholes equations. Although the theoretical convergence results are not provided in this paper, several numerical examples are performed to verify the efficiency of the approach, and it is expected that the approach could be extended to solve more complex free boundary value problems arising in either physics or finance.

\section{References}

Brunner, H. (1985). The numerical solution of weakly singular Volterra integral equations by collocation on graded meshes. Math. Comp., 45: 417-437.

Brunner,H. (2004). Collocation Methods for Volterra Integral and Related Functional Equations. Cambridge University Press, Cambridge.

Cox, J., Ross, S. and Rubinstein, M. (1979). Option pricing: A simplified approach. J. Finan. Econom., 7: 229-263.

Detemple, J. and Tian, W. (2002). The valuation of American options for a class of diffusion processes. Managemant Sci., 48: 917-937.

Fusai, G., Recchioni, M.C. (2007). Analysis of quadrature methods for pricing discrete barrier options. J. Econom. Dynam. Control, 31: 826-860.

Han, H. and Wu, X. (2003). A fast numerical method for the Black-Scholes equation of American options. SIAM J. Numer. Anal., 41: 2081-2095.

Huang, J., Subrahmanyam,M. and Yu. G. (1996). Pricing and hedging American options: A recursive integration method. Rev. Financial Stud., 9: 277-330.

Hull, J. (2007). Fundamentals of Futures and Options Markets, Prentice Hall.

Jiang, L. (2005). Mathematical Modeling and Methods of Option Pricing. World Scientific Press, Singapore.

$\mathrm{Ju}$, N. (1998). Pricing an American option by approximating its early exercise boundary as a multipiece exponential function. Rev. Financial Stud., 11: 627-646.

Khaliq, A., Voss, D.A. and Kazmi, K. (2008). Adaptive $\theta$-methods for pricing American options. J. Comput. Appl. Math., 222: 210-227.

Kim, I.J. (1990). The analytic valuation of American options. Rev. Finan. Stud., 3:547-572.

Landau, H.G. (1950). Heat conduction in a melting solid. Quart. J. Appl. Math., 8: 81-94.

Robinstein, M. (1991). One of another. Risk 4: 30-32.

Tangman, D.Y., Gopaul, A. and Bhuruth,M.(2008). A fast high-order finite difference algorithm for pricing American options. J. Comput. Appl. Math., 222: 17-29. 
Wong, H. and Zhao, J. (2008). An artificial boundary method for American option pricing under the CEV model. SIAM J. Numer. Anal., 46: 2183-2209.

Table 1. Testing the limit of $\tilde{\mathrm{S}}_{f}(t)$ when $t \rightarrow \infty$ for Example 4.1

\begin{tabular}{|c|l|l|l|l|l|c|}
\hline $\mathrm{t}$ & 750 & 760 & 770 & 780 & 790 & 800 \\
\hline$\tilde{\mathrm{S}}_{f}(t)$ & 3.260222 & 3.260028 & 3.20017 & 3.260119 & 3.2599984 & 3.259923 \\
\hline
\end{tabular}

Table 2. Testing the limit of $\tilde{\mathrm{S}}_{f}(t)$ when $t \rightarrow \infty$ for Example 4.2

\begin{tabular}{|c|l|l|l|l|l|c|}
\hline $\mathrm{t}$ & 450 & 460 & 470 & 480 & 490 & 500 \\
\hline$\tilde{\mathrm{S}}_{f}(t)$ & 26.795730 & 26.795084 & 26.794588 & 26.794266 & 26.794059 & 26.793956 \\
\hline
\end{tabular}

Table 3. Testing the limit of $\tilde{\mathrm{S}}_{f}(t)$ when $t \rightarrow \infty$ for Example 4.2

\begin{tabular}{|c|l|l|l|l|l|c|}
\hline $\mathrm{t}$ & 750 & 760 & 770 & 780 & 790 & 800 \\
\hline$\tilde{\mathrm{S}}_{f}(t)$ & 3.260222 & 3.260028 & 3.20017 & 3.260119 & 3.2599984 & 3.259923 \\
\hline
\end{tabular}

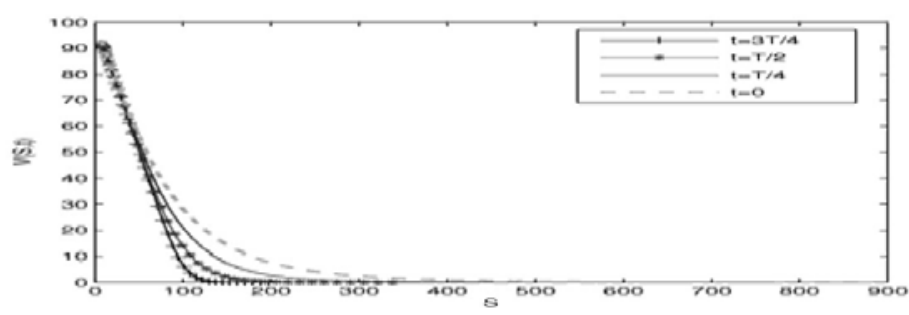

Figure 1. Computational values of underlying assets (2D drawing) for Example 4.1.

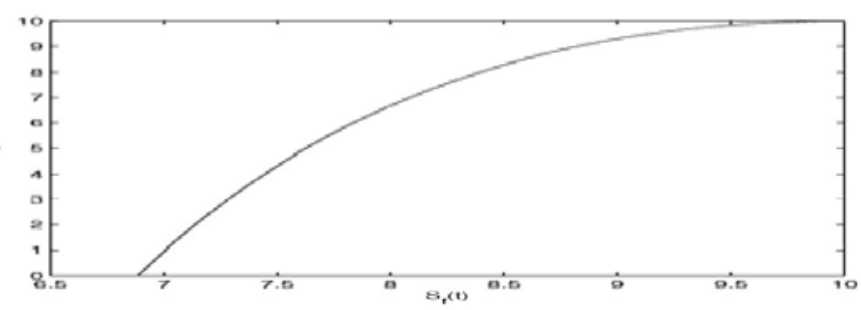

Figure 2. Optimal exercise boundary for Example 4.1.

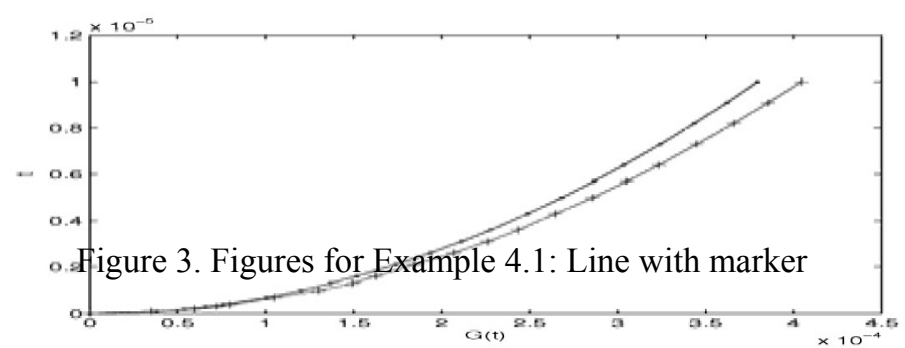

Figure 3. Figures for Example 4.1: Line with marker

$\because$ is for $G(t)=\frac{\frac{r K}{q}-\tilde{S}_{f}(t)}{\frac{r K}{q}}$ and line with marker ' + ' is for $G(t)=\frac{\sigma}{\sqrt{2}} \alpha \sqrt{t}$. 


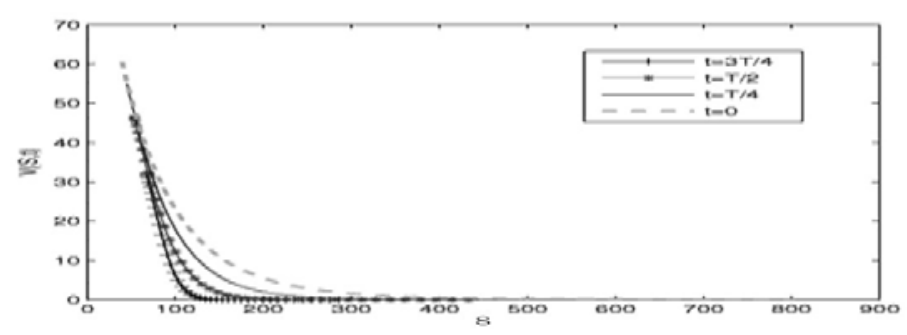

Figure 4. Computational values of underlying assets (2D drawing) for Example 4.2.

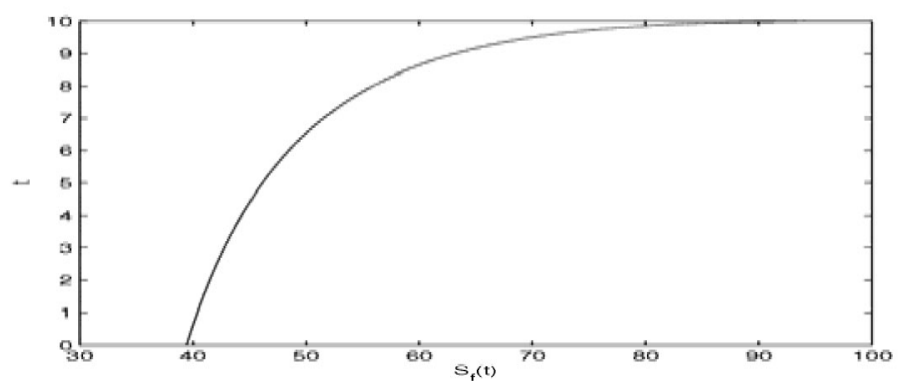

Figure 5. Optimal exercise boundary for Example 4.2.

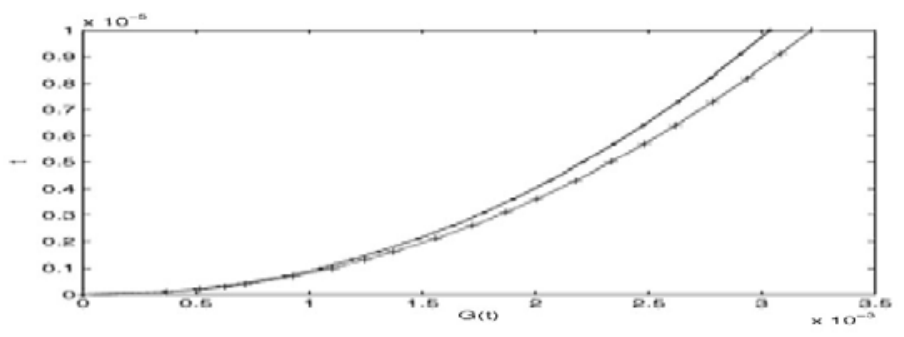

Figure 6. Figures for Example 4.2: Line with marker

', is for $G(t)=\frac{\mathrm{K}-\tilde{S}_{f}(t)}{\mathrm{K}}$ and line with marker ' + ' is for $G(t)=\sqrt{2} \sigma \sqrt{t|\ln t|}$.

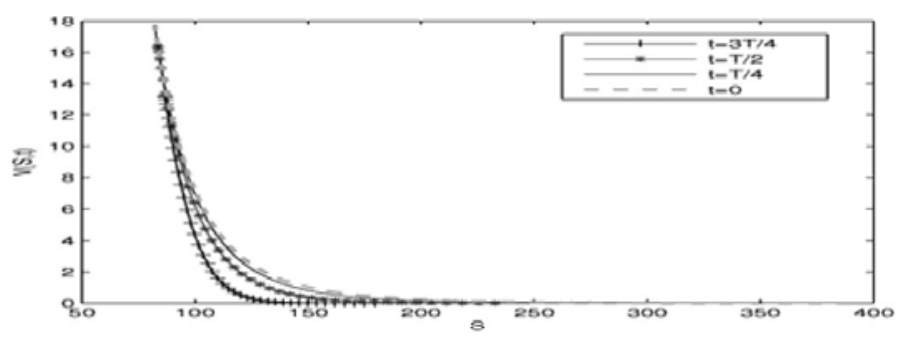

Figure 7. Computational values of underlying assets (2D drawing) for Example 4.3. 


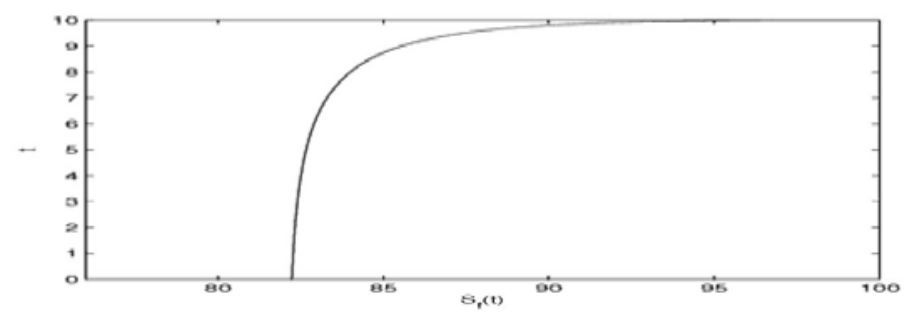

Figure 8. Optimal exercise boundary for Example 4.3.

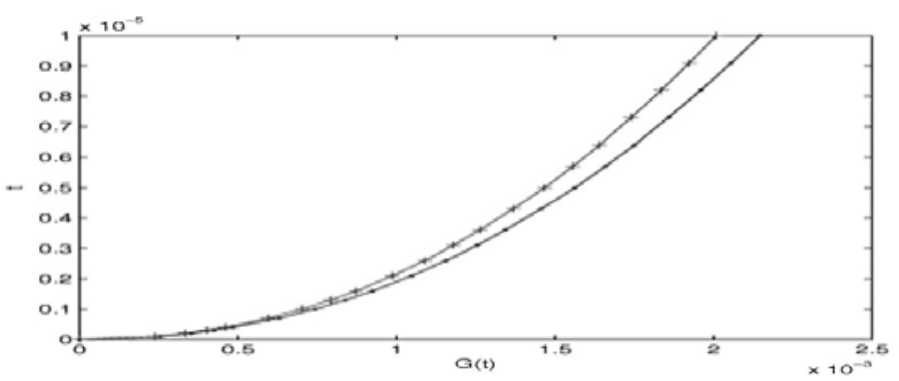

Figure 9. Figures for Example 4.3: Line with marker

'? is for $G(t)=\frac{\mathrm{K}-\tilde{S}_{f}(t)}{\mathrm{K}}$ ) and line with marker '+' is for $G(t)=\sigma \sqrt{t|\ln t|}$. 